\title{
2. ORGANIZACIÓN DE LA ATENCIÓN A LA SALUD MENTAL
}

\section{Planes de Salud Mental}

En el Estado Español, las CCAA iniciaron el proceso de reforma de la atención a la salud mental partiendo de la Ley General de Sanidad y del Informe de la Comisión Ministerial para la reforma psiquiátrica (4). En ambos documentos se proponía el modelo comunitario como el modelo a seguir, modelo que había sido alentado por la Organización Mundial de la Salud (OMS).

En la actualidad, todas las CCAA tienen un Plan de Salud Mental aprobado, la mayoría en la década de los 90 (tabla 2). Casi todos han sido revisados excepto en Aragón, Baleares, Extremadura, Navarra y La Rioja.

Tabla 2. Planificación

\begin{tabular}{|l|c|c|l|}
\hline $\begin{array}{l}\text { COMUNIDAD } \\
\text { AUTONOMA }\end{array}$ & $\begin{array}{c}\text { PLAN SALUD } \\
\text { MENTAL }\end{array}$ & $\begin{array}{c}\text { APROBADO } \\
\text { ANNO }\end{array}$ & REVISADO \\
\hline ANDALUCIA & SI & - & PLAN INTEGRAL 2003 - 2007 \\
\hline ARAGON & SI & 2002 & NO \\
\hline ASTURIAS & SI & 1998 & PLAN ESTRATÉGICO 2001 - 2003 \\
\hline BALEARES & SI & 1998 & N0 \\
\hline CANARIAS & SI & 1997 & 2003 \\
\hline CANTABRIA & SI & 1998 & 2002 \\
\hline CASTILLA LA MANCHA & SI & 1984 & 1993 \\
\hline CASTILLA LEON & SI & 1991 & 2002 \\
\hline CATALUÑA & SI & 1991 & $1996-1999$ \\
\hline EUSKADI & SI & 1983 & 1990 \\
\hline EXTREMADURA & SI & 1996 & N0 \\
\hline GALICIA & SI & 1987 & $1997-2003$ \\
\hline MADRID & SI & $1989-91$ & 2003 (ambos) 1997: PASPEMGC: CONSEJERÍA DE SERVICIOS SOCIALES \\
\hline MELILLA & SI & - & - \\
\hline MURCIA & SI & 1995 & 1999 \\
\hline NAVARRA & SI & 1986 & N0 \\
\hline RIOJA & SI & 1999 & N0 \\
\hline VALENCIA (2002) & SI & 1991 & 2001 \\
\hline
\end{tabular}


Hemos de destacar que sólo Andalucía ha elaborado un Plan Integral (20032007); Asturias ha elaborado un Plan Estratégico (2001-2003). En Euskadi se han realizado varios documentos técnicos (1990), siendo el segundo de ellos un Plan Estratégico. Madrid tiene un Plan de Salud Mental 2003-2008 de la Consejería de Sanidad y el Plan de Atención Social al Enfermo Mental Grave y Crónico de la Consejería de Servicios Sociales.

Pensamos que es importante reseñar que, aunque todas las CCAA tienen ya un Plan de Salud Mental aprobado, no existe un Servicio de Planificación específico para Salud Mental en: Cantabria, Melilla, La Rioja y Valencia. (tabla 3)

Respecto a la Salud Mental Infanto-Juvenil, sólo Andalucía y Murcia tienen un Plan específico de Salud Mental Infanto-Juvenil. En Castilla La Mancha, dicho Plan se ha incluido en el General.

Tabla 3. Servicio de Planificación

\begin{tabular}{|c|c|}
\hline COMUNIDAD AUTÓNOMA & SERVICIO PLANIFICACIÓN SALUD MENTAL \\
\hline ANDALUCIA & Programa Autonómico de salud mental \\
\hline ARAGON & $\mathrm{SI}$ \\
\hline ASTURIAS & $\mathrm{SI}$ \\
\hline BALEARES & SI \\
\hline CANARIAS & $\mathrm{SI}$ \\
\hline CANTABRIA & NO \\
\hline CASTILLA- LA MANCHA & SI \\
\hline CASTILLA LEON & SI \\
\hline CATALUÑA & $\mathrm{SI}$ \\
\hline EUSKADI & $\mathrm{SI}$ \\
\hline EXTREMADURA & SI. Subdirección de Salud Mental \\
\hline GALICIA & $\mathrm{SI}$ \\
\hline MADRID & $\mathrm{SI}$ \\
\hline MELILLA & NO \\
\hline MURCIA & $\mathrm{SI}$ \\
\hline NAVARRA & $\mathrm{SI}$ \\
\hline RIOJA & NO \\
\hline VALENCIA (2002) & NO \\
\hline
\end{tabular}

\section{Normativa: Leyes, Decretos y Órdenes}

El desarrollo y aplicación de los Planes de Salud Mental ha conllevado la implementación de nuevas normativas. Algunas de estas normativas han surgido a partir de disposiciones nacionales y otras se han configurado desde la situación político-administrativa de cada CA. De todas ellas destacamos: 
- Leyes

Canarias:

Ley 11/1994 de Ordenación Sanitaria.

Cantabria:

Ley $1 / 1996$ de salud mental.

Castilla la Mancha: Ley 8/2000 de Ordenación Sanitaria.

Castilla León: $\quad$ Ley 1/1993 de Ordenación del Sistema Sanitario.

Euskadi:

Galicia:

Ley 8/1997 de Ordenación Sanitaria.

Ley 8/1991 de creación del Servicio Galego de Saúde.

Ley 9/1991 de Medidas básicas para la inserción social, modificada por la Ley $1 / 1999$.

Madrid:

Ley 12/2001 de Ordenación sanitaria de la Comunidad Autónoma de Madrid.

Valencia: $\quad$ Ley 8/1987 de creación del Servicio Valenciano de Salud.

- Decretos

Andalucía:

Asturias:

Decreto 338/88 de Organización de los Servicios de Salud Mental Decreto 81/1986 sobre Organización y funcionamiento de los servicios de salud mental. Modificado por los Decretos 119/1991, 61/1994, 183/1995 y 40/1997.

Baleares: $\quad$ Decreto 37/2001 de creación del Fòrum de Salut Mental. Decreto 29/2002 de estructura orgánica del Servei Balear de Salut Decreto 34/2002 que modifica el Decreto 86/2000, que modifica los Decretos 86/2000 y 192/1996 de organización del Servicio Balear de Salud (SERBASA) y Gestión Sanitaria de Mallorca (GESMA).

Decreto 99/2002 de Ordenación de la atención a la Salud Mental.

Canarias: Decreto 83/1998 de Creación Consejos Insulares de Rehabilitación Psicosocial y Acción Comunitaria

Cantabria: Decreto 46/1998 de organización y funcionamiento de la asistencia psiquiátrica y salud mental.

Castilla León: $\quad$ Decreto 83/1989 donde se regula la organización y funcionamiento de los servicios de salud mental y asistencia psiquiátrica.

Cataluña: Decreto Ley 1999 sobre la Red de Centros de Salud Mental de utilización pública.

Galicia:

Decreto 389/1994 por el que se de regula la salud mental.

Decreto 58/1996 de transferencia a la Comunidad Autónoma del Complejo Hospitalario Provincial-Rebullón, dependiente de la Diputación Provincial de Pontevedra.

Decreto 135/1998 por el que se integra el Hospital Psiquiátrico Provincial 0 Rebullón en el Complejo Hospitalario Xeral-Cíes de Vigo. 
Madrid:

Decreto 347/2002por el que se regulan los pisos protegidos, viviendas de transición y unidades residenciales para personas con trastornos mentales persistentes.

Decreto 122/1997 de Servicios Sociales de atención Social, Rehabilitación y soporte comunitario de enfermos mentales graves y crónicos.

Decreto 1/2002 de creación de la Oficina Regional de Coordinación de Salud Mental.

Navarra: $\quad$ Decreto 293/1998 de Transformación del Hospital Psiquiátrico en Centro Psicogeriátrico.

Valencia: $\quad$ Decreto 148/1986: Prestación Servicios en materia de salud mental. Decreto 132/1996: Necesidades de los enfermos mentales.

Decreto 81/1998: Definición recursos dirigidos a salud mental y asistencia psiquiátrica.

\section{- Órdenes}

Baleares:

Orden 11/4/2002 de funciones de la Conselleria de Salut i Consum. Castilla la Mancha: Orden 15/10/85: Guía Unidad de Salud Mental Infanto-Juvenil. Castilla León: Orden 9/3/90 que regula la estructura de coordinación en materia de salud mental y asistencia psiquiátrica.

Orden 19/4/91 que establece la delimitación territorial de la asistencia psiquiátrica.

Galicia: $\quad$ Orden 2/11/86 por la que se crea la Comisión Asesora en materia de salud mental, modificada por la Orden del 2/10/96.

Orden 20/11/1989 por la que se reorganizan los Centros de Diagnóstico y Orientación Terapéutica en Unidades de Salud Mental Infanto-Juvenil.

Orden 21/11/1989 por la que se adscriben funciones al Centros de Salud Mental de A Coruña.

Orden de 30/5/1990 de creación del programa de formación posgraduada de Psicólogos para la atención de la salud mental. Orden 29/1/1996 por la que se regula la ordenación de los dispositivos de salud mental en la provincia de Ourense.

Orden 12/5/1998 por la que se regula la ordenación de los dispositivos de salud mental de la provincia de Pontevedra.

Orden 15/7/1998 por la que se crea la Comisión Asesora en Psicogeriatría.

Orden 23/10/1998 por la que regula los centros de rehabilitación psicosocial y laboral para enfermos mentales crónicos como desarrollo de los dispositivos de apoyo comunitario. 
Orden 20/4/1999 sobre atribución a las funciones definidas en el artículo $3.2^{\circ}$ del Decreto 389/1994 por el que se regula la salud mental en Galicia.

Orden 24/1/2001 por la que se regulan las ayudas individuales no periódicas para minusválidos y personas mayores.

Orden $1 / 2 / 2001$ por la que se establecen programas de fomento de empleo en empresas de economía social, para la promoción de empleo autónomo y para la integración laboral de las personas con discapacidad en centros especiales de empleo y trabajo autónomo.

Orden 11/12/2001 por la que se regula el establecimiento de subvenciones para la realización de programas destinados a prestarles atención a los enfermos mentales crónicos en los dispositivos de apoyo comunitario dependientes de las asociaciones de familiares de enfermos psíquicos y otras entidades sin ánimo de lucro. Continuándose con las Órdenes 27/12/2002 y 5/12/2003. Orden $7 / 5 / 2002$ por la que se regulan las ayudas para la realización de actividades de prevención del alcoholismo. Se reafirma con las Órdenes $1 / 8 / 2003$ y 26/12/2003.

Murcia: $\quad$ Orden 13/5/95 de Sectorización de los Servicios Asistenciales de Salud Mental.

\section{Financiación}

Como se observa en la tabla siguiente (tabla 4), las únicas CCAA que poseen una financiación específica para salud mental son: Asturias, Castilla La Mancha, Castilla León, Euskadi, Galicia y Murcia. Hemos de reseñar que en esta Comunidad, el Hospital Psiquiátrico consume el 39,4\% del presupuesto para salud mental.

En Madrid existe una financiación específica para los recursos que no dependen del antiguo INSALUD, básicamente la atención ambulatoria y la rehabilitación. En el momento de este informe, dicha financiación está en proceso de revisión.

Navarra no tiene financiación específica, sin embargo si que tiene un presupuesto anual específico para salud mental.

En Castilla la Mancha, Castilla León y Cataluña no se conoce cual es el presupuesto anual, o como en el caso de Madrid, no se puede calcular al no estar la gestión de los principales proveedores. 
Tabla 4. Financiación

\begin{tabular}{|l|c|c|c|c|}
\hline $\begin{array}{l}\text { COMUNIDAD } \\
\text { AUTÓNOMA }\end{array}$ & ESPECIFICA & $\begin{array}{c}\text { PRESUPUEST0 } \\
\text { ANUAL }\end{array}$ & $\begin{array}{c}\text { \% DEL PRESUPUESTO } \\
\text { TOTAL DE SANIDAD }\end{array}$ & $€ /$ HAB. \\
\hline ANDALUCIA & $\mathrm{N} 0$ & No se sabe & No se sabe & No se sabe \\
\hline ARAGON & $\mathrm{N} 0$ & No se sabe & No se sabe & No se sabe \\
\hline ASTURIAS & $\mathrm{SI}$ & $1.030 .884 .259 €$ & No se sabe & No se sabe \\
\hline BALEARES & $\mathrm{N} 0$ & No se sabe & No se sabe & No se sabe \\
\hline CANARIAS & $\mathrm{N} 0$ & No se sabe & No se sabe & No se sabe \\
\hline CANTABRIA & $\mathrm{N} 0$ & & & \\
\hline CASTILLA LA MANCHA & $\mathrm{SI}$ & No se sabe & No se sabe & No se sabe \\
\hline CASTILLA LEON & $\mathrm{SI}$ & No se sabe & - & - \\
\hline CATALUNA & $\mathrm{SI}$ & No se sabe & 5,48 & 43,87 \\
\hline EUSKADI & $\mathrm{SI}$ & 90,15 mill. $€$ & 7,7 & 44,03 \\
\hline EXTREMADURA & $\mathrm{N} 0$ & $15.000 .000 €$ & $1,5 \%$ & 15 \\
\hline GALICIA & $\mathrm{SI}$ & $107.709 .374 €$ & 5,42 & 39,43 \\
\hline MADRID & $\mathrm{N} 0$ & No se sabe & No se sabe & No se sabe \\
\hline MELILLA & N0 & No se sabe & No se sabe & No se sabe \\
\hline MURCIA & $\mathrm{SI}$ & $2.140 .989 .000 €$ & No se sabe & No se sabe \\
& & *.P.:39,4\% & & \\
\hline NAVARRA & N0 & 23.642 .965 & No se sabe & $42,5 \%$ \\
\hline RIOJA & N0 & No se sabe & No se sabe & No se sabe \\
\hline VALENCIA (2002) & N0 & No se sabe & No se sabe & No se sabe \\
\hline \hline
\end{tabular}

Del presupuesto total de sanidad, sólo conocemos el porcentaje destinado a Salud Mental de las siguientes CCAA:

$\begin{array}{ll}\text { Castilla León } & 5,48 \% \\ \text { Euskadi } & 7,70 \% \\ \text { Extremadura } & 1,50 \% \\ \text { Galicia } & 5,42 \%\end{array}$

Con respecto al porcentaje de euros por habitante dedicado expresamente a la atención a la salud mental, conocemos los siguientes:

$\begin{array}{ll}\text { Cataluña: } & 43,87 € \\ \text { Euskadi: } & 44,03 € \\ \text { Extremadura: } & 15,00 € \\ \text { Galicia: } & 39,43 € \\ \text { Navarra: } & 42,50 €\end{array}$

\section{Gestión de la Red de Salud Mental:}

En todas las CCAA, la atención a la salud mental está integrada en la Sanidad General, tal como recomienda la Ley General de Sanidad. En líneas generales, la gestión se organiza desde las Consejerías de Sanidad. (tabla 5) 
Tabla 5. Gestión de la red de atención a la Salud Mental

\begin{tabular}{|c|c|c|c|}
\hline COMUNIDAD AUTÓNOMA & ADMINISTRACIÓN RESPONSABLE & $\begin{array}{c}\text { INTEGRADA } \\
\text { SANIDAD GENERAL }\end{array}$ & $\begin{array}{l}\text { ESPECÍFICA } \\
\text { SALUD MENTAL }\end{array}$ \\
\hline ANDALUCIA & $\begin{array}{l}\text { Consejería de Salud. } \\
\text { Servicio Andaluz de Salud }\end{array}$ & $\mathrm{SI}$ & NO \\
\hline ARAGON & $\begin{array}{l}\text { Consejería de Salud y Consumo. } \\
\text { Servicio Aragonés de Salud }\end{array}$ & SI & SI \\
\hline ASTURIAS & $\begin{array}{l}\text { Servicio Salud Principado Asturias } \\
\text { Unidad de Coordinación de S.M. }\end{array}$ & $\mathrm{SI}$ & $\mathrm{SI}$ \\
\hline BALEARES & Conselleria de Salut (IB-Salut) & SI & NO \\
\hline CANARIAS & Servicio Canario de Salud & $\mathrm{SI}$ & $\mathrm{SI}$ \\
\hline CANTABRIA & $\begin{array}{l}\text { Conselleria de Sanidad, } \\
\text { Consumo y Servicios Sociales }\end{array}$ & $\mathrm{SI}$ & NO \\
\hline CASTILLA LA MANCHA & $\begin{array}{l}\text { Junta Castilla la Mancha y } \\
\text { Diputación Provincial (local) }\end{array}$ & SI & SI \\
\hline CASTILLA LEON & Consejería Sanidad de Castilla y León & $\mathrm{SI}$ & $\mathrm{SI}$ \\
\hline CATALUÑA & Servei Catalá de Salut & $\mathrm{SI}$ & $\mathrm{SI}$ \\
\hline EUSKADI & Osakidetza (Servicio Vasco de Salud) & $\mathrm{SI}$ & \\
\hline EXTREMADURA & $\begin{array}{l}\text { Servicio Extremeño de Salud. } \\
\text { Diputaciones Provinciales }\end{array}$ & $\mathrm{SI}$ & NO \\
\hline GALICIA & Servicio Gallego de Salud & $\mathrm{SI}$ & $\mathrm{SI}$ \\
\hline MADRID & $\begin{array}{l}\text { Consejería de Sanidad. } \\
\text { Servicio Madrileño de Salud } \\
\text { Consejería de Servicios Sociales }\end{array}$ & $\mathrm{SI}$ & parcialmente \\
\hline MELILLA & $\begin{array}{l}\text { Instituto Nacional de Gestión } \\
\text { Sanitaria INGESA }\end{array}$ & $\mathrm{SI}$ & NO \\
\hline MURCIA & Servicio Murciano de Salud & $\mathrm{SI}$ & $\mathrm{SI}$ \\
\hline NAVARRA & $\begin{array}{l}\text { Consejería de Salud. } \\
\text { Subdirección de Salud Mental }\end{array}$ & $\mathrm{SI}$ & $\mathrm{SI}$ \\
\hline RIOJA & Servicio Riojano Salud & $\mathrm{SI}$ & NO \\
\hline VALENCIA (2002) & $\begin{array}{l}\text { Conselleria de Sanitat, Conselleria } \\
\text { de Benestar Social, Diputación }\end{array}$ & $\mathrm{SI}$ & NO \\
\hline
\end{tabular}

Sin embargo, hemos de señalar que, en algunas CCAA como Cantabria, Castilla la Mancha, Extremadura, Madrid y Valencia participan en esta gestión otras administraciones como las consejerías de servicios sociales y las diputaciones y ayuntamientos.

Es especialmente significativo la participación de la Consejería de Servicios Sociales en la Comunidad Autónoma de Madrid que elaboró, en 1997, un "Plan de Atención Social a personas con enfermedad mental grave y crónica (PASPEMGC)" complementario al Plan de Salud Mental de la Consejería de Sanidad.

Solamente las CCAA de Aragón, Asturias, Canarias, Castilla la Mancha, Castilla León, Cataluña, Galicia, Murcia y Navarra tienen una Gestión específica para Salud Mental, pero desconocemos la infraestructura que conlleva. 


\section{Coordinación de la Red de Salud Mental:}

Una asistencia de calidad en salud mental implica la coordinación entre los distintos dispositivos de la red sanitaria y no sanitaria implicados por un lado, y por otro la coordinación entre las administraciones con alguna responsabilidad en salud mental. Además esta asistencia de calidad implica que estas coordinaciones han de estar apoyadas desde una organización que las regule y garantice, tanto mediante la ordenación de las acciones que deben llevarse a cabo, como la valoración del tiempo de los profesionales dedicado a ellas.

Por todo ello, las preguntas en el Cuestionario sobre el modo de organización de la coordinación en la atención a la salud mental se dividieron en tres items: Unidad Funcional, Coordinación entre Administraciones y Coordinación entre Dispositivos. Los resultados del cuestionario figuran en la tabla 6 de forma detallada. Como datos destacamos:

- Las Unidades Funcionales de Coordinación solamente existen en

- En Castilla la Mancha se denomina: Comisión Consultiva.

- En Galicia, la realiza el Servicio de Salud Mental.

- En Madrid se denomina: Oficina Regional de Coordinación de Salud Mental.

Tabla 6. Coordinación de la Red de Salud Mental

\begin{tabular}{|c|c|c|c|}
\hline $\begin{array}{l}\text { COMUNIDAD } \\
\text { AUTONOMA }\end{array}$ & $\begin{array}{c}\text { ENTRE } \\
\text { ADMINIST. }\end{array}$ & $\begin{array}{l}\text { UNIDAD } \\
\text { FUNCIONAL }\end{array}$ & $\begin{array}{l}\text { REGULACIÓN DE LA COORDIANCIÓN } \\
\text { ENTRE DISPOSITIVOS }\end{array}$ \\
\hline ANDALUCIA & $\mathrm{SI}$ & $\mathrm{SI}$ & SI Junta de Área \\
\hline ARAGON & $\mathrm{SI}$ & $\mathrm{SI}$ & NO \\
\hline ASTURIAS & $\mathrm{SI}$ & NO & $\mathrm{SI}$ \\
\hline BALEARES & $\mathrm{SI}$ & $\mathrm{SI}$ & NO \\
\hline CANARIAS & NO & $\mathrm{SI}$ & $\mathrm{SI}$ \\
\hline CANTABRIA & $\mathrm{SI}$ & NO & NO \\
\hline CASTILLA LA MANCHA & $\mathrm{SI}$ & SI: Comisión Consultiva & $\begin{array}{l}\text { SI: Comité Técnicos de Coordinación } \\
\text { de Área de Salud }\end{array}$ \\
\hline CASTILLA LEÓN & NO & $\mathrm{SI}$ & $\mathrm{SI}$ \\
\hline CATALUÑA & $\mathrm{SI}$ & $\mathrm{SI}$ & $\mathrm{SI}$ \\
\hline EUSKADI & - & - & SI: Comisiones de Salud Mental \\
\hline EXTREMADURA & $\mathrm{SI}$ & NO & SI \\
\hline GALICIA & $\mathrm{SI}$ & Servicio de Salud Mental & $\mathrm{SI}$ \\
\hline MADRID & $\mathrm{SI}$ & $\begin{array}{c}\text { SI: Oficina Regional de } \\
\text { Coordinación de Salud Mental }\end{array}$ & SI: Comisión de Asistencia de Área \\
\hline MELILLA & $\mathrm{SI}$ & $\mathrm{SI}$ & $\mathrm{SI}$ \\
\hline MURCIA & $\mathrm{SI}$ & $\mathrm{SI}$ & $\mathrm{SI}$ \\
\hline NAVARRA & $\mathrm{SI}$ & $\mathrm{SI}$ & $\mathrm{SI}$ \\
\hline RIOJA & $\mathrm{SI}$ & $\mathrm{SI}$ & $\mathrm{SI}$ \\
\hline VALENCIA (2002) & NO & NO & NO \\
\hline
\end{tabular}


- Las coordinaciones entre Administraciones existen en todas las CCAA, excepto en Canarias, Castilla León y Valencia.

- Pensamos que existen en casi todas las CCAA algún tipo de coordinación entre los dispositivos, aunque sólo conocemos que esté regulada formalmente como tal en:

- En Andalucía: se denomina Junta de Área

- Castilla la Mancha: se denomina Comité Técnico de Coordinación de Área de Salud.

- Euskadi: se denominan Comisiones de Salud Mental.

- Madrid: Comisión de Asistencia de Área.

\section{Sistemas de Información:}

Los Sistemas de Información y recogida de datos de las distintas CCAA. Aparecen en la tabla 7. En general el desarrollo de los sistemas de información es insuficiente en la mayoría de las CCAA y muchas veces cada administración responsable tiene su propia metodología de recogida de datos y sistemas de información que no se operan de forma integrada.

Tabla 7. Sistemas de Información

\begin{tabular}{|c|c|c|}
\hline COMUNIDAD AUTÓNOMA & SISTEMA DE INFORMACIÓN & RECOGIDA DE DATOS \\
\hline ANDALUCIA & $\mathrm{SI}$ & $\mathrm{SI}$ \\
\hline ARAGON & En elaboración & $\mathrm{SI}$ \\
\hline ASTURIAS & $\mathrm{SI}$ & $\mathrm{SI}$ \\
\hline BALEARES & NO & NO \\
\hline CANARIAS & SI & $\mathrm{SI}$ \\
\hline CANTABRIA & NO & NO \\
\hline CASTILLA LA MANCHA & Estructura y actividad & General sistema sanitario (AT especializada) \\
\hline CASTILLA LEON & $\mathrm{Sl}$ & $\mathrm{Sl}$ \\
\hline CATALUÑA & SI & $\mathrm{SI}$ \\
\hline EUSKADI & $\mathrm{SI}$ & R.A.C.P. y C. M. B. D. \\
\hline EXTREMADURA & NO & SI \\
\hline GALICIA & SI & R.A.C.P.(implantación parcial), CMBD, SISINFO \\
\hline MADRID & SI & R.A.C.P y Sistemas generales de Atención Especializada: SISCAM, HP-HIS. \\
\hline MELILLA & SI & $\mathrm{SI}$ \\
\hline MURCIA & SI & SI \\
\hline NAVARRA & SI & SI \\
\hline RIOJA & SI & R.A.C.P. \\
\hline VALENCIA (2002) & NO & SI \\
\hline
\end{tabular}

Así, solo se ha informado de la recogida de datos en formato de Registro Acumulativo de Casos Psiquiátricos (R.A.C.P.) en Euskadi, Galicia (implantación parcial), Madrid y la Rioja.

Existen otros sistemas de recogida de datos e información. Además del RACP, el Conjunto Mínimo Básico de Datos (CMBD) se utiliza en Euskadi y Galicia. El SISINFO también en Galicia, y los sistemas de recogida de datos de Atención Especializada (HP-HIS, SISCAM) en Madrid. En Castilla la Mancha, la recogida de datos se realiza dentro del Sistema Sanitario Especializado. 
Baleares y Cantabria no cuentan con un sistema de recogida de datos, ni con un Sistema de Información.

En Aragón se está elaborando el Sistema de Información, que tampoco existe en Extremadura ni Valencia. 